\title{
The interstellar magnetic field near the Galactic center
}

\author{
Katia Ferrière ${ }^{1}$ \\ ${ }^{1}$ Laboratoire d'Astrophysique de Toulouse-Tarbes, Université de Toulouse, CNRS, \\ 14 avenue Edouard Belin, F-31400 Toulouse, France \\ email: ferriere@ast.obs-mip.fr
}

\begin{abstract}
We review the observational knowledge that has built up over the past 25 years on the interstellar magnetic field within $\sim 150 \mathrm{pc}$ of the Galactic center. We also provide a critical discussion of the main observational findings and comment on their possible theoretical interpretations. To conclude, we propose a coherent view of the interstellar magnetic field near the Galactic center, which accounts at best for the vast body of observations.
\end{abstract}

Keywords. ISM: magnetic fields - ISM: general - ISM: structure - (ISM:) cosmic rays - Galaxy: center - Galaxies: magnetic fields

\section{Introduction}

The interstellar medium (ISM) near the Galactic center differs significantly from the ISM in the rest of the Galaxy. The ordinary matter (made of gas and small amounts of dust) tends to be denser, warmer, and more metal-rich, while the magnetic field reaches higher values and has a more poloidal geometry.

The physical characteristics and the spatial distribution of the interstellar gas in the innermost $3 \mathrm{kpc}$ of the Galaxy were reviewed by Ferrière, Gillard, \& Jean (2007). To summarize, the interstellar gas can be found in molecular, atomic, and ionized forms. The molecular gas is globally ten times more abundant than the atomic gas, and together these two neutral components enclose roughly the same amount of mass $\left(\sim 10^{8} M_{\odot}\right)$ as the ionized component. Spatially, the molecular gas tends to concentrate in the so-called central molecular zone (CMZ), a thin sheet parallel to the Galactic plane, which, in projection on the sky, extends out to a radius $r \sim 250 \mathrm{pc}$ at positive longitudes and $r \sim 150 \mathrm{pc}$ at negative longitudes, and has a FWHM thickness $\sim 30 \mathrm{pc}$. The CMZ itself contains a ring-like feature with mean radius $\sim 180 \mathrm{pc}$, now known as the 180-pc molecular ring, and, deeper inside, a population of dense molecular clouds. Outside the CMZ, the molecular gas is confined to a significantly tilted disk, extending in projection out to $r \sim 1.3 \mathrm{kpc}$ on each side of the Galactic center and having a FWHM thickness $\sim 70$ pc. The spatial distribution of the atomic gas is arguably similar to that of the molecular gas, with this difference that the atomic layer is about three times thicker than the molecular layer. In addition, it is likely that the tilted disk encloses proportionally more atomic gas than the CMZ. Finally, the ionized gas is not confined to either the CMZ or the tilted disk; it appears to fill the entire Galactic bulge and to merge smoothly with the ionized gas present in the Galactic disk.

In the present paper, we focus on the interstellar magnetic field in a much smaller region around the Galactic center, which extends only $\sim 300$ pc along the Galactic plane and $\sim 150 \mathrm{pc}$ in the perpendicular direction. Radially, this small region is entirely contained 
within the CMZ. Outside the CMZ, observational data are scanty, so that not much can be said about the magnetic field properties.

In Section 2, we present the observational picture that emerged at the end of the 1980s, and in Section 3, we discuss the main problems inherent in this picture. In Section 4, we describe some new developments arising from a variety of recent observations. Finally, in Section 5, we try to piece everything together into a coherent picture. We also propose theoretical explanations for the existing observations, and we examine how the magnetic field near the Galactic center might connect with the magnetic field in the rest of the Galaxy.

Throughout the paper, the magnetic field vector is denoted by $\vec{B}$, its line-of-sight component by $B_{\|}$, the field strength by $B$, and the Alfvén speed by $V_{\mathrm{A}}$. Besides, $l$ and $b$ denote Galactic longitude and latitude, respectively, $r$ and $z$ denote Galactocentric radial and vertical (i.e., perpendicular to the Galactic plane) coordinates, respectively, and the Sun is assumed to lie at $r_{\odot}=8.5 \mathrm{kpc}$. At this distance from the Galactic center, an angle of $1^{\circ}$ corresponds to a length of $150 \mathrm{pc}$.

\section{The Old Picture}

The first observational clues to the direction and strength of the interstellar magnetic field near the Galactic center date back to the 1980s, when radio-astronomers (starting with Yusef-Zadeh, Morris, \& Chance 1984 and Liszt 1985) discovered systems of radio continuum filaments running nearly perpendicular to the Galactic plane. As summarized by Morris (1996), these filaments are typically a few to a few tens of parsecs long and a fraction of a parsec wide, they appear straight or mildly curved all along their length, and their radio continuum emission is linearly polarized and characterized by a spectral index consistent with synchrotron radiation, hence the denomination of nonthermal radio filaments (NRFs or NTFs).

The long and thin shape of NRFs strongly suggests that they follow magnetic field lines. This suggested alignment is confirmed by the measured radio polarization angles (corrected for Faraday rotation), which indicate that, in the plane of the sky, the magnetic field inside NRFs is indeed oriented along their long axes (e.g., Tsuboi et al. 1985, 1986; Reich 1994; Lang et al. 1999a). From this, it has naturally been concluded that the interstellar magnetic field near the Galactic center is approximately vertical, at least close to the Galactic plane. Farther from the plane, NRFs tend to lean somewhat outwards, consistent with the interstellar magnetic field having an overall poloidal geometry (Morris 1990).

The magnetic field strength inside NRFs is much more uncertain and controversial. The equipartition/minimum-energy field strength is typically $B_{\text {eq }} \sim(50-200) \mu \mathrm{G}$ (Anantharamaiah et al. 1991; LaRosa et al. 2004, and references therein). However, there appears to be no particular reason why NRFs would actually be in an equipartition/ minimum-energy state with cosmic rays. In fact, their apparent rigidity and organized structure suggest instead that they are magnetically dominated (Anantharamaiah et al. 1991; Lang, Morris, \& Echevarria 1999b), so that their actual field strength is probably higher than the equipartition/minimum-energy value.

A completely independent estimate of the magnetic field strength inside NRFs relies on a simple dynamical argument, originally proposed by Yusef-Zadeh \& Morris (1987). According to these authors, the fact that NRFs remain nearly straight, even as they pass through the layer of molecular clouds, suggests that their magnetic pressure, $P_{\mathrm{mag}}$, is higher than the cloud ram pressure, $P_{\text {ram }}$. For a presumably conservative value of $P_{\text {ram }}$, this condition is equivalent to $B \gtrsim 1 \mathrm{mG}$ inside NRFs. 
Going one step further, Morris (1990) argued that NRFs must be pressure-confined. He estimated that the ambient gas pressure, which is dominated by the thermal pressure of the very hot $\left(T \sim 10^{8} \mathrm{~K}\right)$ plasma, is too low by a factor $\sim 30$ to confine NRFs, and he concluded that NRFs must be confined by magnetic pressure. In other words, the $\mathrm{mG}$ magnetic field inferred to exist inside NRFs must also prevail outside.

These considerations led to the notion that a pervasive $\mathrm{mG}$ magnetic field, with an overall poloidal geometry, fills the region containing NRFs - NRFs were initially observed out to $\gtrsim 70$ pc of the Galactic center (Morris 1990), but they are now detected out to $\gtrsim 150$ pc (LaRosa et al. 2004; Yusef-Zadeh, Hewitt, \& Cotton 2004). In this view, NRFs would simply be magnetic flux tubes which happen to be illuminated by the injection of synchrotron-radiating electrons (Morris 1990).

\section{Problems with a Pervasive mG Magnetic Field}

The dynamical argument leading to $B \gtrsim 1 \mathrm{mG}$ inside NRFs presents several shortcomings: (1) Not all the NRFs remain nearly straight. Some clearly display severe distortions (e.g., the so-called Snake; Gray et al. 1995), while others could have deformations that escape detection from Earth because of projection effects. (2) Although most NRFs pass through the layer of molecular clouds, it is likely that only a fraction of them are actually colliding with clouds. (3) Even for truly colliding NRFs, the condition $P_{\text {mag }} \gtrsim P_{\text {ram }}$ is probably too stringent. Unless they collide at more than one location along their length, a more appropriate condition would be $V_{\mathrm{A}} \gg v_{\text {cloud }}$ (Chandran 2001) or, equivalently, $B \gg 10 \mu \mathrm{G}$.

The pressure-balance argument leading to $B \sim 1 \mathrm{mG}$ in the general ISM raises even more serious objections: (1) The thermal pressure of the very hot plasma, $P_{\text {hot }}$, might be significantly higher than estimated by Morris (1990). According to the X-ray spectroscopic results of Koyama et al. (1996), $P_{\text {hot }}$ could be comparable to the pressure of a $1 \mathrm{mG}$ magnetic field, and hence high enough to confine NRFs. (2) NRFs could also be confined by magnetic tension forces (Lesch \& Reich 1992; Uchida \& Guesten 1995), as suggested by the helical fields detected in or around some NRFs (e.g., Yusef-Zadeh \& Morris 1987; Gray et al. 1995). (3) More fundamentally, NRFs do not need to be confined at all; they could very well be transient or dynamic structures out of mechanical balance with their surroundings. For instance, Boldyrev \& Yusef-Zadeh (2006) argued that turbulence in the Galactic center region naturally leads to a highly intermittent magnetic field distribution, with strongly magnetized filaments arising in an otherwise weak-field background, and they suggested that the turbulent filaments could correspond to the observed NRFs. Alternatively, Shore \& LaRosa (1999) proposed that NRFs are the long and thin magnetic wakes produced by a weakly magnetized Galactic wind impinging on molecular clouds near the Galactic center.

Aside from the potential flaws in the dynamical and pressure-balance arguments, a magnetic field as strong as $1 \mathrm{mG}$ implies synchrotron lifetimes that might be too short to explain certain observations (see, e.g., Yusef-Zadeh 2003; Morris 2007). At the radio frequencies (typically $1.5 \mathrm{GHz}$ and $5 \mathrm{GHz}$ ) of most NRF observations, the synchrotron lifetimes in a $1 \mathrm{mG}$ field are only $\sim 2 \times 10^{4} \mathrm{yr}\left(2.7 \times 10^{4} \mathrm{yr}\right.$ at $1.5 \mathrm{GHz}$ and $1.5 \times 10^{4} \mathrm{yr}$ at $5 \mathrm{GHz}$ ). If the synchrotron-radiating electrons are injected somewhere along the NRFs (for instance, at the site of interaction with a molecular cloud) and if they stream away along the NRFs at about the Alfvén speed, then, over their lifetimes, they travel distances $\sim(10-60)$ pc, which might be too short to account for the length of the longest NRFs. At the lower radio frequencies $(74 \mathrm{MHz}$ and $330 \mathrm{MHz}$ ) of the diffuse nonthermal emission detected by LaRosa et al. (2005) (see Section 4), the synchrotron lifetimes in a $1 \mathrm{mG}$ field 
are somewhat longer, but still only $\sim 10^{5} \mathrm{yr}\left(1.2 \times 10^{5}\right.$ yr at $74 \mathrm{MHz}$ and $0.6 \times 10^{5} \mathrm{yr}$ at $330 \mathrm{MHz}$ ), which the authors considered to be "shorter than any plausible replenishment timescale".

Finally, a $1 \mathrm{mG}$ magnetic field filling the $\sim(300 \mathrm{pc})^{2} \times 150 \mathrm{pc}$ region over which the $\mathrm{NRF}$ phenomenon is observed (LaRosa et al. 2004) encloses $\sim 10^{55}$ ergs in magnetic energy. This huge amount of magnetic energy corresponds to the energy released by $\sim 10^{4}$ supernova explosions. It is roughly comparable to the kinetic energy associated with Galactic rotation in the CMZ, while being significantly larger than the kinetic energy associated with turbulent motions in the same region. It is also larger than, or comparable to, the thermal energy of the very hot plasma present in the Galactic center region. What the origin of such a huge magnetic energy could be remains unclear.

\section{New Developments}

The picture of a poloidal $\mathrm{mG}$ magnetic field pervading the central $300 \mathrm{pc}$ of the Galaxy is further challenged by a variety of more recent observations, which we now review.

Yusef-Zadeh et al. (2004) put together a catalog of all the (well-established + likely candidate) NRFs detected at $1.5 \mathrm{GHz}$, and they presented a schematic diagram of their spatial distribution in the plane of the sky. Their diagram conveys a sense that the vast majority of NRFs are indeed nearly straight (with the notable exception of the Snake) and that they tend to align with the vertical. In reality, however, only the longer NRFs strictly follow this tendency; the shorter NRFs exhibit a broad range of orientations, with only a loose trend toward the vertical.

Lower-frequency $(74 \mathrm{MHz}$ and $330 \mathrm{MHz}$ ) radio imaging of the Galactic center region by LaRosa et al. (2005) revealed a $6^{\circ} \times 2^{\circ}$ source of diffuse nonthermal (presumably synchrotron) emission. The inferred minimum-energy field strength (on spatial scales $\gtrsim$ $5 \mathrm{pc})$ is $\simeq(6 \mu \mathrm{G})(k / f)^{2 / 7}$, where $k$ is the cosmic-ray proton-to-electron energy ratio and $f$ the filling factor of the synchrotron-emitting gas. Both parameters are quite uncertain, but we may reasonably assume that $k$ lies somewhere between the two canonical values 1 (generally adopted in the vicinity of powerful cosmic-ray sources) and 100 (generally adopted in the Galactic disk), while $f \gtrsim 0.01$. The minimum-energy field strength must then lie in the range $\simeq(6-80) \mu \mathrm{G}$.

More recently, Crocker et al. (2010) found that the spectrum of the diffuse radio emission from the Galactic center region exhibits a downward break at $\sim 1.7 \mathrm{GHz}$, which can be explained by the synchrotron-radiating electrons undergoing a transition from bremsstrahlung to synchrotron cooling. The measured break frequency imposes a relationship between magnetic field strength (which governs synchrotron cooling) and gas density (which governs bremsstrahlung cooling), and this relationship makes it possible, for any given value of $B$, to model the cooled electron distribution as well as its $\gamma$-ray (inverse-Compton + bremsstrahlung) emission. Requiring that the latter do not exceed the $300 \mathrm{MeV} \gamma$-ray emission measured by EGRET then leads to the constraint $B \gtrsim$ $50 \mu \mathrm{G}$.

$$
* * * * *
$$

Far-infrared/submillimeter polarization studies of dust thermal emission enable one to probe the direction (in the plane of the sky) of the interstellar magnetic field inside dense molecular clouds. As a general rule, far-infrared polarimetry applies to the warmer parts of molecular clouds, while submillimeter polarimetry applies to their colder parts.

Davidson (1996), who reviewed the existing far-infrared polarization measurements toward dense regions located within $\sim 100 \mathrm{pc}$ of the Galactic center, noted that the 
measured magnetic field direction is generally roughly parallel to the Galactic plane. She argued that this field direction could be explained by the dense gas moving relative to the surrounding diffuse gas and either distorting the local poloidal field or dragging its own distorted field from another Galactic location.

The magnetic field direction inferred from far-infrared polarimetry is largely backed up by submillimeter polarization observations. In particular, the $450 \mu \mathrm{m}$ polarization map of Novak et al. (2003), which covers a $170 \mathrm{pc} \times 30 \mathrm{pc}$ area around the Galactic center, confirms that the magnetic field threading molecular clouds is, on the whole, approximately parallel to the Galactic plane. To reconcile the horizontal field measured in molecular clouds with the poloidal field traced by NRFs, Novak et al. (2003) proposed that the large-scale magnetic field near the Galactic center is predominantly poloidal in the diffuse ISM and predominantly toroidal in dense regions along the Galactic plane, where it was sheared out in the azimuthal direction by the differential rotation of the dense gas.

The conclusions of Novak et al. (2003) were refined by Chuss et al. (2003), who found that the measured magnetic field direction depends in fact on the molecular gas density, being generally parallel to the Galactic plane in high-density regions and generally perpendicular to it in low-density regions. According to their preferred interpretation, the large-scale magnetic field near the Galactic center was initially poloidal everywhere, but in dense molecular clouds, where the gravitational energy density exceeds the magnetic energy density, it became sheared out into a toroidal field by the clouds' motions. In the framework of this scenario, Chuss et al. (2003) estimated a characteristic field strength $\sim 3 \mathrm{mG}$ inside molecular clouds, by assuming that clouds where the field is half-way between toroidal and poloidal are those where gravitational and magnetic energy densities are equal.

Near-infrared polarization observations of starlight absorption by dust also offer a promising tool to trace the magnetic field direction (again in the plane of the sky) in dense regions near the Galactic center. The recent $50 \mathrm{pc} \times 50 \mathrm{pc}$ near-infrared polarization map of Nishiyama et al. (2009) exhibits a strong tendency for the polarization vectors to align with the Galactic plane, in good agreement with the results of far-infrared/submillimeter polarimetry. However, this map shows no hint of a potential correlation between field direction and gas density.

$$
* * * * *
$$

Zeeman splitting of radio (atomic or molecular) spectral lines offers a direct means of measuring the line-of-sight component of the magnetic field, $B_{\|}$, in dense, neutral regions. $\dagger$ So far, unfortunately, Zeeman splitting measurements have only yielded mixed results.

For the circumnuclear disk, the $\sim 10$ pc sized innermost molecular region, Killeen, Lo, \& Crutcher (1992) derived $B_{\|} \sim-2 \mathrm{mG}$ both in the southern part (firm detection) and in the northern part (marginal detection). A little later, Marshall, Lasenby, \& Yusef-Zadeh (1995) obtained only an upper limit $\sim 0.5 \mathrm{mG}$ in each of the northern and southern parts, whereas Plante, Lo, \& Crutcher (1995) reported 7 detections (1 positive and 6 negative values of $B_{\|}$) ranging between $-4.7 \mathrm{mG}$ and $+1.9 \mathrm{mG}$ toward the northern part. These disparate Zeeman results are not necessarily contradictory; they can be reconciled if $B_{\|}$ varies substantially (especially if $B_{\|}$changes sign) across the circumnuclear disk.

Farther away from the Galactic center, Crutcher et al. (1996) measured values of $B_{\|}$ ranging between $\simeq-0.1 \mathrm{mG}$ and $-0.8 \mathrm{mG}$ toward the Main and North cores of Sgr B2.

$\dagger$ By convention in the Zeeman splitting community, a positive (negative) value of $B_{\|}$corresponds to a magnetic field pointing away from (toward) the observer. 
In contrast, Uchida \& Guesten (1995) reported only non-detections, with $3 \sigma$ upper limits $\sim(0.1-1) \mathrm{mG}$, toward 13 selected positions within a few degrees of the Galactic center (including Sgr B2).

The mixture of positive detections, with $\left|B_{\|}\right| \sim(0.1-1) \mathrm{mG}$, and non-detections, with $\left|B_{\|}\right| \lesssim(0.1-1) \mathrm{mG}$, outside the circumnuclear disk can probably be attributed partly to possible dilution of the Zeeman signal by averaging over the observed area and along the line of sight and partly to genuine differences in the local values of $B_{\|}$. Genuine differences in $B_{\|}$are expected if the magnetic field inside molecular clouds is roughly horizontal, as indicated by far-infrared/submillimeter polarization observations, and has random directions in the Galactic plane. Zeeman results then suggest that the total field strength inside molecular clouds is roughly $\sim 1 \mathrm{mG}$, with a possible range from a few $0.1 \mathrm{mG}$ to a few $\mathrm{mG}$. This broad range encompasses the characteristic field strength $\sim 3 \mathrm{mG}$ estimated from submillimeter polarimetry (Chuss et al. 2003).

$$
* * * * *
$$

Faraday rotation measures (RMs), for their part, provide information on $B_{\|}$in the diffuse ionized medium. $\dagger$ Both positive and negative RMs have been obtained toward the Galactic center, with typical values ranging from a few hundred to a few thousand rad $\mathrm{m}^{-2}$ (e.g., Tsuboi et al. 1985; Yusef-Zadeh \& Morris 1987; Gray et al. 1995; Lang et al. 1999a, 1999b). In principle, if the line-of-sight depth of the Faraday-rotating screen and the free-electron number density within it are known, $B_{\|}$can be inferred from the measured RMs. In practice, though, both parameters are difficult to estimate, partly because the Faraday-rotating screen itself is often hard to locate. Nevertheless, with the parameter values adopted in the existing RM studies, the measured RMs generally translate into $B_{\|} \sim \pm$ a few $\mu \mathrm{G}$ (e.g., Tsuboi et al. 1985; Yusef-Zadeh \& Morris 1987; Gray et al. 1995).

The values of $\left|B_{\|}\right|$inferred from RM measurements are compatible with both a dynamically dominant magnetic field $\sim 1 \mathrm{mG}$ and a minimum-energy field $\simeq(6-80) \mu \mathrm{G}$. It is, however, noteworthy that if the large-scale magnetic field in the diffuse ISM near the Galactic center has a poloidal geometry, with only a small component along the line of sight, the low end of the minimum-energy range might be difficult to reconcile with $\left|B_{\|}\right| \sim$ a few $\mu \mathrm{G}$.

Examination of the measured RM signs has led to contradictory conclusions. Novak et al. (2003), who collected all the available RMs toward NRFs within $1^{\circ}$ of the Galactic center, brought to light a definite pattern in the sign of RM, such that RM $>0$ in the quadrants $(l>0, b>0)$ and $(l<0, b<0)$ and $\mathrm{RM}<0$ in the quadrants $(l>0, b<0)$ and $(l<0, b>0)$. This pattern, they explained, could result from azimuthal shearing by the Galactic differential rotation of an initially vertical magnetic field pointing north (dense molecular clouds, which are confined close to the Galactic plane, tend to rotate faster than the diffuse gas).

In contrast, Roy, Rao, \& Subrahmanyan (2005), who derived the RMs of 60 background extragalactic sources through a $12^{\circ} \times 4^{\circ}$ window centered on the Galactic center, obtained mostly positive values, with no evidence for a sign reversal with latitude or with longitude. Roy, Rao, \& Subrahmanyan (2008) pointed out that this predominance of positive RMs is consistent with either the large-scale Galactic magnetic field having a bisymmetric spiral configuration or the magnetic field near the Galactic center being oriented along the Galactic bar.

$\dagger$ The convention for the sign of $B_{\|}$in the Faraday rotation community is opposite to that adopted in the Zeeman splitting community. Here, a positive (negative) value of $B_{\|}$corresponds to a magnetic field pointing toward (away from) the observer. 


\section{Conclusions}

Based on the critical observational overview presented in the preceding sections, we (tentatively) propose that the interstellar magnetic field near the Galactic center has the following properties:

(1) In the diffuse intercloud medium, the magnetic field is approximately poloidal on average. There exist a number of localized filamentary structures (the so-called NRFs), wherein the field is almost certainly above equipartition with cosmic rays $\left[B_{\text {eq }} \sim(50-\right.$ 200) $\mu \mathrm{G}$ ] and could, in some cases, be as strong as $B \gtrsim 1 \mathrm{mG}$. Outside NRFs, the field is almost certainly weaker, although not necessarily by a huge factor, as there is some evidence pointing to $B \gtrsim 50 \mu \mathrm{G}$; one may not rule out the possibility that such a field could be in equipartition with cosmic rays $\left[B_{\text {eq }} \simeq(6-80) \mu \mathrm{G}\right]$.

(2) In dense molecular clouds, the magnetic field is approximately horizontal. The field strength is probably quite high, with typical values ranging between a few $0.1 \mathrm{mG}$ and a few $\mathrm{mG}$.

The approximately poloidal direction of the magnetic field in the diffuse intercloud medium can be explained by various scenarios, including inward advection from the Galactic disk (Sofue \& Fujimoto 1987; Chandran, Cowley, \& Morris 2000), outflows from the Galactic nucleus (Sofue 1984; Chevalier 1992), and a local dynamo. The reason why the magnetic field within dense molecular clouds is approximately horizontal can be understood if the poloidal intercloud field was sheared out in a horizontal direction by the cloud bulk motions (from differential rotation or from turbulence) with respect to the diffuse intercloud medium (Novak et al. 2003; Chuss et al. 2003) or by the forces (of compressive or tidal nature) that created and/or shaped the clouds (Morris \& Serabyn 1996, and references therein). Alternatively, it is possible that the field within molecular clouds became decoupled from the intercloud field (Morris \& Serabyn 1996; Morris 2007), for instance, as a result of cloud rotation.

If the magnetic field near the Galactic center is approximately poloidal in the diffuse intercloud medium, it is most likely antisymmetric. $\dagger$ This inferred antisymmetry is fully supported by the set of RMs collected by Novak et al. (2003), which displays a clear sign reversal across the midplane (see Section 4). RMs also indicate that the magnetic field runs counterclockwise above the midplane and clockwise below it. If this configuration results from azimuthal shearing by the Galactic differential rotation, then the vertical field must be pointing north $\left(B_{z}>0\right)$. Interestingly, these directions of the azimuthal and vertical field components coincide with those thought to prevail in the inner Galactic halo (Han, Manchester, \& Qiao 1999; Mao et al. 2010).

Hence, the magnetic field near the Galactic center could possibly be a natural continuation of the magnetic field in the inner halo. The combined field would evidently be antisymmetric. However, its poloidal component would not be a pure dipole (as often assumed in the cosmic-ray propagation community), otherwise $B_{z}$ would have opposite signs along the rotation axis and at the position of the Sun. Instead, it seems more likely that the combined Galactic-center + inner-halo field would have a poloidal component pointing everywhere north, together with a (possibly strong) azimuthal component produced by Galactic shear.

$\dagger$ A magnetic field is said to be symmetric (antisymmetric) in $z$, or, equivalently, quadrupolar (dipolar), if its horizontal component is an even (odd) function of $z$ and its vertical component an odd (even) function of $z$. 


\section{References}

Anantharamaiah, K. R., Pedlar, A., Ekers, R. D., \& Goss, W. M. 1991, MNRAS, 249, 262

Boldyrev, S. \& Yusef-Zadeh, F. 2006, ApJ, 637, L101

Chandran, B. D. G. 2001, ApJ, 562, 737

Chandran, B. D. G., Cowley, S. C., \& Morris, M. 2000, ApJ, 528, 723

Chevalier, R. A. 1992, ApJ, 397, L39

Chuss, D. T., Davidson, J. A., Dotson, J. L., et al. 2003, ApJ, 599, 1116

Crocker, R. M., Jones, D. I., Melia, F., Ott, J., \& Protheroe, R. J. 2010, Nature, 463, 65

Crutcher, R., Roberts, D. A., Mehringer, D. M., \& Troland, T. H. 1996, ApJ, 462, L79

Davidson, J. A. 1996, in ASP Conf. Ser., 97, Polarimetry of the Interstellar Medium, ed. W. G. Roberge \& D. C. B. Whittet (San Francisco: ASP), 504

Ferrière, K., Gillard, W., \& Jean, P. 2007, A\&A, 467, 611

Gray, A. D., Nicholls, J., Ekers, R. D., \& Cram, L. E. 1995, ApJ, 448, 164

Han, J. L., Manchester, R. N., \& Qiao, G. J. 1999, MNRAS, 306, 371

Killeen, N. E. B., Lo, K. Y., \& Crutcher, R. 1992, ApJ, 385, 585

Koyama, K., Maeda, Y., Sonobe, T., et al. 1996, PASJ, 48, 249

Lang, C. C., Anantharamaiah, K. R., Kassim, N. E., \& Lazio, T. J. W. 1999a, ApJ, 521, L41

Lang, C. C., Morris, M., \& Echevarria, L. 1999b, ApJ, 526, 727

LaRosa, T. N., Brogan, C. L., Shore, S. N., et al. 2005, ApJ, 626, L23

LaRosa, T. N., Nord, M. E., Lazio, T. J. W., \& Kassim, N. E. 2004, ApJ, 607, 302

Lesch, H. \& Reich, W. 1992, A\&A, 264, 493

Liszt, H. S. 1985, ApJ, 293, L65

Mao, S. A., Gaensler, B. M., Haverkorn, M., Zweibel, E. G., Madsen, G. J., McClure-Griffiths,

N. M., Shukurov, A., \& Kronberg, P. P. 2010, ApJ, 714, 1170

Marshall, J., Lasenby, A. N., \& Yusef-Zadeh, F. 1995, MNRAS, 274, 519

Morris, M. 1990, in IAU Symp., 140, Galactic and Intergalactic Magnetic Fields, ed. R. Beck, R. Wielebinski \& P. P. Kronberg (Dordrecht: Kluwer), 361

Morris, M. 1996, in IAU Symp., 169, Unsolved Problems of the Milky Way, ed. L. Blitz \& P. J. Teuben (Dordrecht: Kluwer), 247

Morris, M. 2007, ArXiv Astrophysics e-prints

Morris, M. \& Serabyn, E. 1996, ARAA, 34, 645

Morris, M., Uchida, K., \& Do, T. 2006, Nature, 440, 308

Nishiyama, S., Tamura, M., Hatano, H., et al. 2009, ApJ, 690, 1648

Novak, G., Chuss, D. T., Renbarger, T., et al. 2003, ApJ, 583, L83

Plante, R. L., Lo, K. Y., \& Crutcher, R. 1995, ApJ, 445, L113

Reich, W. 1994, in NATO ASI, 445, The Nuclei of Normal Galaxies: Lessons from the Galactic Center, ed. R. Genzel \& A. Harris (Dordrecht: Kluwer), 55

Roy, S., Rao, A. P., \& Subrahmanyan, R. 2005, MNRAS, 360, 1305

Roy, S., Rao, A. P., \& Subrahmanyan, R. 2008, A\& $A, 478,435$

Serabyn, E. \& Morris, M. 1994, ApJ, 424, L91

Shore, S. N. \& LaRosa, T. N. 1999, ApJ, 521, 587

Sofue, Y. 1984, PASJ, 36, 539

Sofue, Y. \& Fujimoto, M. 1987, PASJ, 39, 843

Tsuboi, M., Inoue, M., Handa, T., Tabara, H., \& Kato, T. 1985, PASJ, 37, 359

Tsuboi, M., Inoue, M., Handa, T., et al. 1986, AJ, 92, 818

Uchida, K. I. \& Guesten, R. 1995, A\&A, 298, 473

Yusef-Zadeh, F. 2003, ApJ, 598, 325

Yusef-Zadeh, F., Hewitt, J. W., \& Cotton, W. 2004, ApJS, 155, 421

Yusef-Zadeh, F. \& Morris, M. 1987, ApJ, 322, 721

Yusef-Zadeh, F., Morris, M., \& Chance, D. 1984, Nature, 310, 557 


\section{Discussion}

ZWEIBEL: Is the cosmic-ray density in the NRFs higher than ambient?

FERRIÈRE: We don't know for sure. The cosmic-ray density cannot be measured directly. It can be constrained, to some extent, by the measured synchrotron intensity, but the constraints depend on the assumed magnetic field strength. If the magnetic field is roughly uniform throughout the medium (as argued by Morris 1990), then the relativistic-electron density, and presumably also the cosmic-ray density, must be higher in NRFs. In this case, NRFs can be regarded as magnetic flux tubes illuminated by a local injection of relativistic electrons. In contrast, if the magnetic field is stronger inside NRFs (as suggested by all the problems inherent in the uniform-field picture; see Section 3), which most likely supposes that NRFs are compressed flux tubes, then two antagonistic effects may come into play. On the one hand, when an NRF forms by compression, the attached cosmic rays are compressed together with the field lines and their density increases. On the other hand, once cosmic rays find themselves inside a high- $B \mathrm{NRF}$, they stream away along field lines (at about the Alfvén speed) and escape into the halo faster than cosmic rays in the ambient medium, so that their density decreases relative to the ambient cosmic-ray density. In addition, relativistic electrons cool off (via synchrotron radiation) more rapidly than in the ambient medium.

ToOmRE: Could you expand upon what may be the origin of the lengthy filaments near the center of our Galaxy, which has long been described as the "violent center of our Galaxy" (aside from the supermassive black hole Sgr A*)?

FERRIÈRE: Different scenarios have been put forward to explain the origin of the lengthy radio filaments (the so-called NRFs) near the Galactic center. A first possibility is that NRFs result from a local injection of relativistic electrons. For instance, when a molecular clump moves across the ambient magnetic field lines, magnetic reconnection may occur at its leading edge and lead to particle acceleration (Serabyn \& Morris 1994). Another possibility is that NRFs result from a local compression or shearing of magnetic field lines. Here, two possible candidates are turbulence, which, in the Galactic center region, is expected to produce strongly magnetized filaments (Boldyrev \& Yusef-Zadeh 2006), and a Galactic wind impinging on molecular clouds, which could generate long magnetic wakes behind the clouds (Shore \& LaRosa 1999).

BRANDENBURG: Is anything known about the orientation of the helical structures and their position relative to the midplane (above/below)? Also, the slightly different directions of the filaments might be explicable by them having different distances to the observer.

FERRIÈre: Observational evidence has been found for the existence of helical magnetic fields winding about some of the radio filaments, including the filaments of the Radio Arc (e.g., Yusef-Zadeh \& Morris 1987; Gray et al. 1995; Morris, Uchida, \& Do 2006). In none of these cases is the evidence very strong, and the putative helical fields are not perceptible over more than two or three wavelengths. The orientation of the helical structures and their position relative to the midplane can be determined in individual cases, but there are not enough cases to infer a general trend of orientation versus position above/below the midplane.

Regarding the second part of the question, I think the slightly different directions of the NRFs is more likely explained by turbulence. 